\title{
A Latent Approach to Understanding Pain in Nursing Home Residents Who are Unable to Self-Report Pain
}

\author{
Sarah Forrester (D) \\ Attah Mbrah \\ Kate L Lapane (D) \\ Division of Epidemiology, Department of \\ Population and Quantitative Health \\ Sciences, University of Massachusetts \\ Medical School, Worcester, MA, USA
}

Context: Pain assessment in people with cognitive impairment is challenging.

Objective: The study sought to 1) identify pain subgroups based on staff-assessed pain, agitated and reactive behavior, functional status, and symptoms of depression; and 2) understand if cognitive impairment was associated with transitions between pain subgroups at nursing home admission, 3 months, and 6 months.

Methods: Using national Minimum Data Set 3.0 data (2011-2016), we included 26,816 newly admitted residents with staff-assessed pain at admission, 3 months, and 6 months. Pain subgroups were identified by latent class analysis at each time point. Transitions between pain subgroups were described using latent transition analysis.

Results: Five latent statuses of pain were identified at admission: "Behavioral and Severe Depression" (prevalence stable, severe or worsening cognitive impairment: 11\%, mild/ moderate or improved cognitive impairment: 10\%), "Functional" $(21 \% ; 25 \%)$, "Physical" $(22 \% ; 23 \%)$, "Behavioral" (23\%, 19\%), and "Low" (23\%; 24\%). Regardless of change in cognitive status, most residents remained in the same pain latent class. Among residents with stable, severe or worsening cognitive impairment, $11 \%$ in the "Behavioral" class transitioned to the "Behavioral and Severe Depression" class by 3 months. Fewer residents transitioned between latent classes in the 3- to 6-month period ( $>80 \%$ remained in their 3-month class). Conclusion: For nursing home residents unable to self-report pain, consideration of additional indicators including functioning, depressive symptoms, and agitation may be useful in identifying pain subgroups. Longitudinal changes in the pain subgroups over 6 months post-admission highlight that residents with severe cognitive impairment may be at risk for worsening pain.

Keywords: latent transition analysis, dementia, depressive symptoms, agitation, long-term care

\section{Introduction}

In nursing homes, residents commonly experience pain. ${ }^{1}$ Despite similar frequencies of painful conditions across levels of cognitive impairment, residents with worse cognitive function have decreased pain documented in nursing homes. ${ }^{2}$ Among residents with moderate to severe dementia, $45 \%$ experienced moderate to severe pain at some point in the previous week. ${ }^{3}$ Yet, nursing home direct care staff may fail to recognize pain in residents unable to self-report pain owing to dementia ${ }^{4}$ or may confuse indicators of pain with behavioral and psychological symptoms of dementia. ${ }^{5}$

Pain assessment in people with cognitive impairment is challenging, as alterations in speech/language and memory often worsen as disease progresses. ${ }^{6}$
Correspondence: Kate L Lapane

Division of Epidemiology, Department of Population and Quantitative Health Sciences, University of Massachusetts Medical School, Worcester, MA, USA

Email Kate.Lapane@umassmed.edu 
Observational pain scales have been developed for use in people with cognitive impairment such as the Pain Assessment in Advance Dementia (PAINAD), ${ }^{7}$ visual analog scales, ${ }^{8}$ the Discomfort in Dementia Scale and Protocol, ${ }^{9,10}$ the checklist of non-verbal pain indicators, ${ }^{11}$ and proxy pain questionnaires. ${ }^{12}$ Among patients who are unable to self-report pain, facial expressions and behavioral symptoms are often included in scales designed to assess pain. ${ }^{13}$ Restlessness, rubbing, guarding, rigidity, and physical aggression show strong evidence as body movement indicators for pain while bracing, decreased mobility, flinching, and pacing show moderate evidence. ${ }^{14}$ The assessment of pain in those with dementia is further complicated by the welldocumented relationships between pain and depression ${ }^{15}$ and dementia and depression. ${ }^{16}$ Among those who are unable to self-report pain, disentangling pain and depression can be challenging.

Pain affects quality of life, ${ }^{17}$ mental health, ${ }^{18}$ and activities of daily living. ${ }^{17}$ Understanding the nontraditional ways that pain may be exhibited and the relationship between pain and depression symptoms is important to ensure adequate treatment. This study sought to identify pain subgroups among residents unable to self-report pain and to understand if cognitive impairment was associated with movement between pain subgroups at admission, 3 months, and 6 months among residents. We hypothesized that 1) pain would be expressed through non-verbal sounds, vocal complaints of pain, facial expressions, protective body movements, behavioral symptoms, and that the relationship of pain to depression and functional status would result in distinct pain subgroups and 2) the level of cognitive impairment would affect how residents move between subgroups at 3- and 6-months post admission.

\section{Methods}

This study was approved by the University of Massachusetts Medical School Institutional Review Board (IRB ID: H00011964). In accordance with our Data Use Agreement with the Centers for Medicare and Medicaid Services, the Minimum Data Set resource used in the study complies with data protection and privacy regulations.

\section{Conceptual Framework Guiding Research}

Our study was guided by the seminal multidimensional pain model, which conceptualized pain as the result of a complex interaction between physiological, emotional, cognitive, and behavioral domains. ${ }^{19}$ Pain is a multidimensional construct with sensory, behavioral, emotional, and cognitive components. ${ }^{20}$ The dominant theories regarding pain are applicable to those with dementia. ${ }^{21}$ We have modified the model as the original model views mood as an outcome and we will consider it a symptom in our analyses. ${ }^{22}$

\section{Data Source}

The Minimum Data Set (MDS) 3.0 contains information on comprehensive assessments of the health and functional status of every nursing home resident in Medicare/ Medicaid certified nursing homes in the United States. $^{23,24}$ Most US nursing homes are Medicare/ Medicaid certified ( $96 \%)$. The MDS assessment includes demographic information, diagnosed chronic conditions, self-reported depressive symptoms, staffassessed pain, cognitive function, and behavioral and functional symptoms. A comprehensive MDS assessment is completed at admission. Thereafter, a subset of items is collected on quarterly assessments. ${ }^{25}$ The MDS is completed by multiple members of the care team, ${ }^{26}$ and includes information on self-reported and staff-observed symptoms and behaviors. ${ }^{23,27}$ All participant assessments were completed in-person at the nursing home.

\section{Sample Selection}

We identified 1,222,705 skilled nursing facilities or longstay residents aged $\geq 65$ years newly admitted to US nursing homes between 2011 and 2016, without a cancer diagnosis, non-comatose, and having 3- and 6-month assessments (Figure 1). Newly admitted residents were selected because they could be compared to each other in relation to time since admission. Residents with cancer were excluded because their pain was likely different from those with nonmalignant pain. Our study focused on residents unable to self-report pain. The MDS manual provides explicit instructions on whether a staff assessment for pain should be conducted; staff assessment occurs only if the self-reported pain assessment interview is not completed. ${ }^{24}$ The study sample included 26,816 residents.

\section{Measures}

Staff assessment of pain was carried out based on information from the 5 days preceding the admission assessment (restricted to time in the nursing home) through the MDS Pain Behavior Scale. ${ }^{28}$ Staff assessment of four behavioral pain indicators included review of medical records, interviews with staff, and direct observation. The four pain 


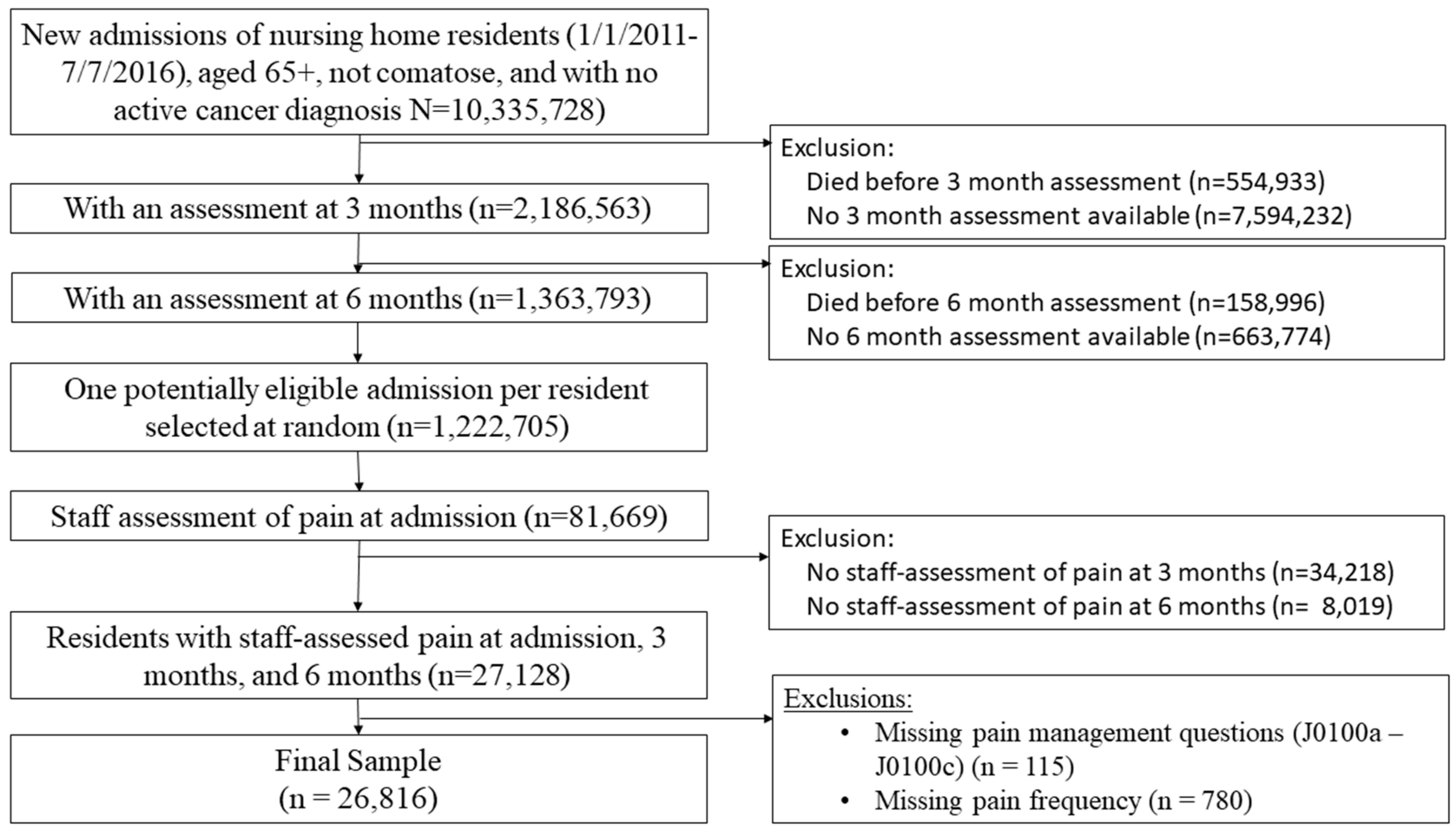

Figure I Flowchart of the construction of the analytic sample.

behaviors were 1) nonverbal sounds (eg, crying, whining, groaning); 2) vocal complaints (eg, ouch, stop); 3) facial expressions (eg, grimaces, winces); and 4) protective body movements (eg, bracing, rubbing, clutching a body part during movement). Medical records were reviewed, and information was confirmed with the direct care staff working most closely with the resident during activities of daily living (across all shifts). The direct care staff were asked about the presence of each pain indicator. Direct observation was conducted during care activities. The average agreement (kappa) for staff observed pain behaviors was between 0.94 and $0.96 .^{28}$ The frequency of pain behaviors within the past 5 days was documented (ie, 1-2 days, 3-4 days, daily). We calculated Cronbach's alpha as 0.63 in our sample. Pain was dichotomized as absent (none of the signs were observed or documented in the last 5 days) or present (any of the indicators of pain were present in the last 5 days.

The 10-item Patient Health Questionnaire Observational Version (PHQ-9 OV) ${ }^{29}$ and adaptation of the original PHQ- $9^{30,31}$ were used to screen for depression among residents who could not complete an interview (range: 0-30). Cronbach's alpha for the PHQ-9 in primary care was $0.89 .^{32}$ We calculated Cronbach's alpha at 0.71 for the PHQ9-OV in our sample. We only used data from participants who were unable to self-report the PHQ-9. Anhedonia, hopelessness, insomnia/hypersomnia, poor appetite/overeating, worthlessness, impaired concentration, and psychomotor agitation/retardation, but not suicidal ideation $(<1 \%)$ were included.

Functional status was assessed with the activities of daily living (ADL) hierarchy scale ${ }^{33}$ based on personal hygiene, toilet use, locomotion, and eating, which adapts the common measurements of $\mathrm{ADLs}^{34,35}$ to the MDS dataset. Cronbach's alpha was 0.94 in the MDS validation study and 0.85 in our sample. The scale is scored $0-6$ with 0 indicating independence in all four ADLs, 3 indicating at least extensive help needed in personal hygiene or toilet use, and 6 indicating total dependence in all four ADLs. We dichotomized the scale $(1=$ dependence (scores of 5 or 6); $0=$ independent through extensive assistance).

The Agitated and Reactive Behavior Scale ${ }^{36}$ is a composite measure of physical and verbal behavioral symptoms directed at other people, other behavioral symptoms not directed at other people (eg, disrobing, pacing), and rejection of care that was updated from the original Aggressive Behavior Scale. ${ }^{37}$ Cronbach's alpha in the validation study for newly admitted residents was 0.71 and 0.73 in our sample. The ARBS score range is 0 (behavior not exhibited on any item) to 12 (behavior 
occurs daily on all 4 items), but we dichotomized the scale ( $1=$ any behavioral symptoms present, $0=$ no behavioral symptoms present).

The Cognitive Function Scale (CFS), a measure of cognitive status based on MDS items, ${ }^{27,38}$ integrates information from the Brief Interview for Mental Status ${ }^{39}$ (score range 0 -15 , lower score indicates worse cognition, 3 items total) and the Cognitive Performance Scale $^{40}$ (score range 0-6, higher score indicates worse cognition, 5 items total). The internal consistency (Cronbach's alpha) of the CPS was 0.70 in a validation study. ${ }^{41}$ The CFS classifies residents as intact (able to complete BIMS and scored 13-15), mildly impaired (8-12 BIMS score or 0-2 CPS score), moderately impaired (07 BIMS score or 3-4 CPS score, or severely impaired (could not complete BIMS and 5-6 CPS score). The scale has four items total and a score range of $0-6$, but we created a dichotomized variable that indicated change in cognitive impairment from admission to 6 months. Residents who had severe cognitive impairment at nursing home admission and who remained severely impaired at 6 months were included in the "worse" category.

\section{Analyses}

Descriptive statistics characterized the sample overall and by change in level of cognitive impairment. To identify pain subgroups and understand how cognitive impairment affects resident transition between pain groups, we used latent transition analysis (LTA), a longitudinal extension of latent class analysis (LCA). ${ }^{42,43}$ LTA includes a measurement component and a structural component. The first step in the analysis is to complete a crosssectional LCA at each time point (ie, admission, 3 months, and 6 months). LCA produces posterior probabilities or the likelihood of a resident endorsing an item given their membership in a class which makes up the measurement component. The structural component consists of the class prevalence at each time point and the conditional probability of transition from one class to another at each time point given the class membership at the previous time point.

Separate LCAs were completed to determine the ideal number of classes at admission, 3 months, and 6 months. Twelve dichotomous indicators measuring pain, behavioral symptoms, ADLs, and depression (interest, feeling down, sleep, energy, appetite, feeling like a failure, concentration, psychomotor retardation, and temper) were included in each model. Fit indices (AIC, BIC, Lo-Mendell Rubin [LMR], and Bootstrapped Likelihood Ratio [BLRT] tests $)^{44}$ were obtained for models with classes ranging from 2 to 8 for each time point. Next, LTA was used to measure transition probabilities from admission to 3 months to 6 months. We ran an overall LTA with the full sample and an LTA in which change in cognitive status was used as a grouping variable to influence the transitions.

\section{Results}

\section{Sample Characteristics}

Table 1 shows that most were aged $\geq 75$ years and the sample majority was unmarried, White, and women. More than $90 \%$ had moderate to severe cognitive impairment as measured by the CFS. Fifty-two percent of residents had either stable severe cognitive impairment or worsened cognitive impairment over the 6 months since nursing home admission.

\section{Latent Class Analyses at Nursing Home Admission, 3 Months, and 6 Months}

Separate LCAs at admission, 3 months, and 6 months were examined to determine the optimal number of classes at each time point. We examined the bivariate residuals to check the assumption of conditional independence. Conditional independence assumes that all indicators are independent of one another after conditioning on the latent class. We found that the indicators for feeling like a failure and psychomotor retardation remained dependent after conditioning on the latent class and neither indicator distinguished between latent classes, so we removed them as indicators. The final LCA included 10 indicators. The AIC and BIC indicated a six-class solution at each time point while the BLRT and LMR indicated a five-class solution at each time point. We selected the five-class solution because six-class solution did not add pertinent information to the model. Figure 2 displays the indicators and their conditional probabilities within each class. The "Low" class had probabilities of 0.20 or less for all indicators. The "Behavioral and Severe Depression" class showed high probability of behavioral symptoms as well as high probabilities of all depression symptoms. The "Behavioral" class also had high probability of behavioral symptoms but only a high probability of being shorttempered or easily annoyed, a behavioral symptom of depression. The "Functional" class had a high probability of being dependent or totally dependent while the "Physical" class had a high probability of being dependent 
Table I Descriptive Characteristics of Nursing Home Residents with Staff Assessed Pain, 20I I-20I6, by Change in Cognitive Status

\begin{tabular}{|c|c|c|c|}
\hline Admission & $\begin{array}{c}\text { Sample } \\
(n=26,8 \mid 6)\end{array}$ & $\begin{array}{l}\text { Stable, Severe Cognitive } \\
\text { Impairment or Worsened in } \\
6 \text { Months }(n=5035)\end{array}$ & $\begin{array}{l}\text { Stable, Mild/Moderate Cognitive } \\
\text { Impairment or Improved in } 6 \\
\text { Months }(n=21,78 I)\end{array}$ \\
\hline \multicolumn{4}{|l|}{ Age, years } \\
\hline $65-74$ & 17.7 & 17.0 & 17.9 \\
\hline $75-84$ & 38.0 & 37.9 & 38.0 \\
\hline$\geq 85$ & 44.3 & 45.1 & 44.0 \\
\hline Women & 72.4 & 72.3 & 72.4 \\
\hline \multicolumn{4}{|l|}{ Marital Status } \\
\hline Married & 32.6 & 33.9 & 32.3 \\
\hline \multicolumn{4}{|l|}{ Race } \\
\hline Non-White & 22.4 & 20.5 & 22.9 \\
\hline \multicolumn{4}{|l|}{ Symptoms (Yes) } \\
\hline Pain & 35.9 & 38.7 & 35.3 \\
\hline Dependent in Activities of Daily Living ${ }^{a}$ & 47.2 & 44.1 & 48.0 \\
\hline $\mathrm{ARBS}^{\mathrm{b}}$ & 35.4 & 38.1 & 34.9 \\
\hline Little interest or pleasure in doing things ${ }^{c}$ & 18.3 & 17.2 & 18.3 \\
\hline Feeling down, depressed ${ }^{c}$ & 20.9 & 23.6 & 20.2 \\
\hline Trouble falling or staying asleep ${ }^{c}$ & 19.4 & 20.4 & 19.2 \\
\hline Feeling tired, having little energyc ${ }^{c}$ & 28.7 & 30.2 & 28.4 \\
\hline Decreased/increased appetite ${ }^{c}$ & 20.4 & 20.1 & 20.4 \\
\hline Trouble concentrating ${ }^{c}$ & 35.0 & 34.5 & 35.2 \\
\hline Being short tempered/easily annoyed ${ }^{c}$ & 20.8 & 24.4 & 20.2 \\
\hline
\end{tabular}

Notes: ${ }^{a}$ Activities of Daily Living measured by the ADL Hierarchy Scale (dependent and totally dependent versus all others); ${ }^{b}$ Agitated and Reactive Behavior Scale (any behavioral symptom); 'Depression symptoms measured by Patient Health Questionnaire (PHQ-9) Depression Scale (self-assessed and staff assessed).

or totally dependent and a high probability of feeling tired or having little energy, a physical depression symptom. Based on the initial LCA, we conducted the LTA with five classes and 10 indicators.

\section{Latent Transitions from Nursing Home Admission to 6 Months}

We examined transitions across classes from admission to 6 months. We ran both an overall LTA and an LTA with change in cognitive status (same/better versus worse) as a grouping variable so that we could examine the rates of transition across cognitive status groups. Among the full sample, the estimated prevalence of each class was similar at each time point. The only class that appeared to increase was the "Behavioral and Severe Depression" class which had estimated prevalence of $10 \%, 13 \%$, and $14 \%$ at admission, 3 months, and 6 months, respectively. Class stability over time was similar within cognitive change groups (Table 2). The "Behavioral and Severe Depression" class increased from admission to 3 months and then stabilized for both groups. All other classes had similar prevalence at each time point. The main difference in class membership between groups can be seen in the differences in class membership in the "Low" class and the "Functional" class. Among those whose cognitive status did not worsen, about $30 \%$ are in the "Low" class compared to about $19 \%$ among those whose cognitive status did worsen or remained severe. Conversely, about $29 \%$ of those whose cognitive status worsened or remained severe were in the "Functional" class compared to about $19 \%$ in those whose cognitive status remained mild or moderate or improved.

Among the full sample, there was less stability from admission to month 3 than from month 3 to month 6 (Table 3). About 11\% of the sample transitioned from the "Behavioral and Severe Depression" to "Physical" and $12 \%$ transitioned from "Behavioral and Severe Depression" to "Behavioral". Similarly, 12\% transitioned from "Physical" to "Functional" and 11\% transitioned from "Behavioral" to "Low". Class membership was more stable from 3 months to 6 months; class stability ranged from $90 \%$ for the "Physical" and "Low" classes to 95\% for the "Functional" class.

Group-specific transitions followed a similar pattern. There was more movement from admission to 3 months 


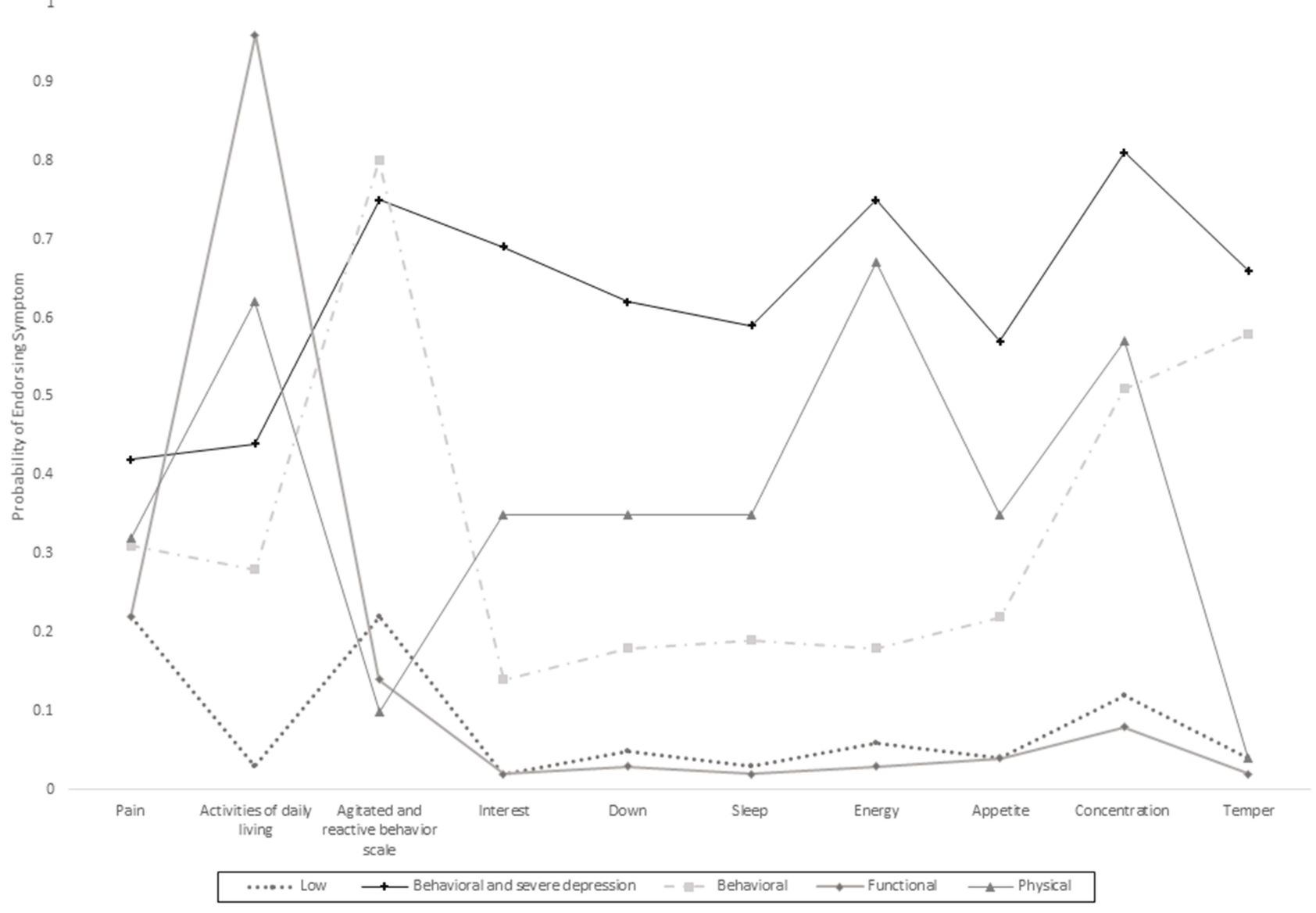

Figure 2 Item response probabilities for four-class solution.

and more stability from 3 to 6 months. From admission to 3 months, there were multiple transitions of 0.10 or higher among both cognitive change groups. Several transitions were similar between the two groups, such as transitions from the "Behavioral and Severe Depression" class to the "Behavioral" class, and from the "Behavioral and Severe Depression" class to the "Physical" class. Specific to the same/better cognitive change group, there were higher proportions of participants moving to the "Low" class from other classes $(13 \%-14 \%)$. Among the stable severe cognitive impairment/worsening cognitive change group, only the "Behavioral" class had a higher proportion that moved to the "Low" class (10\%). Unique to the stable severe cognitive impairment/worsening cognitive change

Table 2 Estimated Class Membership Across Time Points by 6-Month Change in Cognitive Impairment Among US Nursing Home Residents with Staff-Assessed Pain (20II-20I6)

\begin{tabular}{|c|c|c|c|c|c|}
\hline & Behavioral and Severe Depression & Functional & Physical & Behavioral & Low \\
\hline \multicolumn{6}{|c|}{ Stable, Severe Cognitive Impairment or Worsened in 6 Months Since Admission } \\
\hline Admission & $11 \%$ & $21 \%$ & $22 \%$ & $23 \%$ & $23 \%$ \\
\hline Three Months & $15 \%$ & $21 \%$ & $22 \%$ & $21 \%$ & $23 \%$ \\
\hline Six Months & $15 \%$ & $21 \%$ & $22 \%$ & $21 \%$ & $22 \%$ \\
\hline \multicolumn{6}{|c|}{ Stable, Mild/Moderate Cognitive Impairment or Improved in 6 Months Since Admission } \\
\hline Admission & $10 \%$ & $25 \%$ & $23 \%$ & $19 \%$ & $24 \%$ \\
\hline Three Months & $14 \%$ & $24 \%$ & $23 \%$ & $19 \%$ & $24 \%$ \\
\hline Six Months & $14 \%$ & $24 \%$ & $23 \%$ & $19 \%$ & $23 \%$ \\
\hline
\end{tabular}




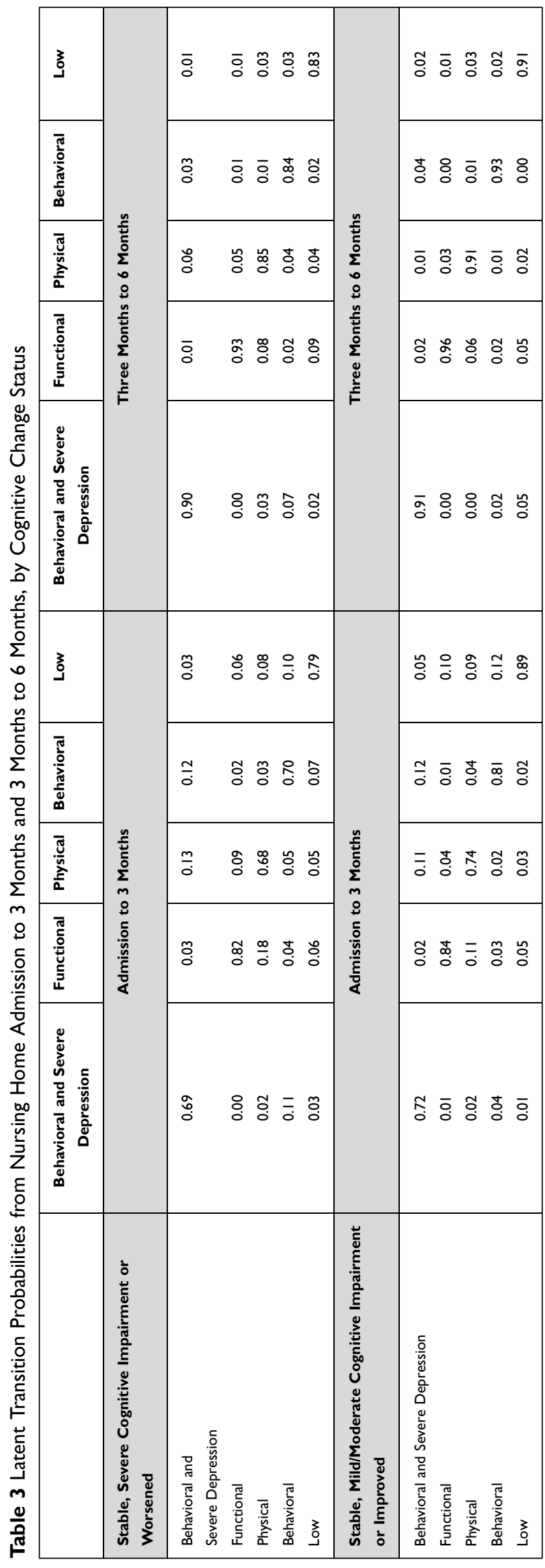

group was the $16 \%$ that moved from "Physical" to "Functional". Each represents a transition to less severe symptoms. For example, transitioning from the "Physical" class to the "Functional" class indicates that the symptom of feeling tired or having no energy lessened. Similarly, transitioning from the "Behavioral and Severe Depression" to the "Behavioral" class indicates that the symptoms of severe depression improved and were no longer endorsed. Only residents with stable severe cognitive impairment/ worsening cognitive change had a transition to a more severe class. About $7 \%$ of those in the "Behavioral" class among those with worsening cognition transitioned to the "Behavioral and Severe Depression" class by 3 months, meaning that they "added" severe depression in the 3 months post nursing home admission.

Class stability was more evident from 3 to 6 months post nursing home admission among both groups. Among residents whose cognition stayed the same or improved, there were no transitions higher than 0.06 indicating stability of class membership. Among residents with stable severe cognitive impairment/worsening cognitive change, transitioning from "Behavioral" to "Behavioral and Severe Depression" was attenuated to $4 \%$ and the proportion who transitioned from "Physical" to "Functional" was reduced by half. Residents who had severe, stable or worsening cognition continued in the period from 3 to 6 months to have a higher proportion transitioning into the "Functional" class than those with same/better cognition. Although the transition from "Physical" to "Functional" was attenuated, the proportion of residents who transitioned from "Low" to "Functional" increased by about $3 \%$ from 3 to 6 months compared to the first 3 months post nursing home admission.

\section{Discussion}

We found that consideration of additional measures to detect pain in nursing home residents unable to selfreport pain has value. Among newly admitted nursing home residents unable to self-report pain, there were five pain symptom subgroups differentiated by dependence in ADLs, behavioral symptoms, and symptoms of depression. Three-quarters of residents had symptom profiles aligned with pain symptom subgroups defined by behavioral symptoms (with or without depressive symptoms), function, or physical manifestations. Latent classes were qualitatively similar across time periods (admission, 3 months, 6 months) and change in cognitive impairment status. While most residents remained in the same pain 
symptom subgroup at 3 and 6 months, transitions across subgroups were more apparent in the 3 months post nursing home admission as compared to the 3 to 6 months post admission.

One-third of residents lacking the ability to self-report pain were aligned with latent classes defined by behavioral symptoms [Behavioral and Severe Depression" ( 11$15 \%)$; "Behavioral" subgroups ( 19-23\%)]. Our findings are consistent with previous research supporting the notion that pain may be a root cause of behavioral disturbances as the links between pain, psychiatric symptoms, and depression onset have been shown. ${ }^{45}$ We also found that the prevalence of the "Behavioral and Severe Depression" class increased from admission to 3 months, and 1 in 10 residents with worsening or stable severe cognitive impairment transitioned from the "Behavioral" class to the "Behavioral and Severe Depression" class by 3 months post-admission. There are several plausible explanations for these findings. One explanation is that as nursing home staff get to know residents better over 3 months, documentation of depressive symptoms is more accurate. While no data exist specific to residents unable to self-report pain, previous work has shown little transition between depressive symptom subgroups within the first 3 months of a nursing home stay. ${ }^{46}$ Residents adjusting to their new setting may develop depressive symptoms during the 3-month transitional phase. While a recent study on suicidal ideation in nursing homes noted that the prevalence declined with time in the nursing facility, ${ }^{47}$ research on changes in depressive symptoms throughout the initial transition to nursing home among residents lacking the ability to report pain measures does not exist. Studies to further understand the patterns observed may provide greater direction to improve pain recognition and management in this population. We agree with others who endorse the need to evaluate the triggers of behavioral disturbances and identify appropriate strategies to intervene on modifiable triggers. ${ }^{48}$

Most residents remained in their admission latent class at 3 months andfurther research is neededto understand the reasons why. In a medically supervised setting, we had hoped to see improvements in pain subgroups with time. Use of analgesics and antidepressants ${ }^{49}$ and psychosocial interventions $^{50}$ may reduce pain. Our study was not designed to evaluate the role of pharmacologic interventions. Whether the residents in our study received treatments for pain and/or depression and the extent to which such treatments reduce symptom burden needs to be carefully evaluated in future research.

We had expected greater change in latent classes earlier in the nursing home stay and the data confirmed this expectation. For those transitioning between the latent classes, we observed more change in the first 3 months, with greater stability between 3- and 6-months post nursing home admission. It is well known that the initial period in the nursing home corresponds with major adjustments. ${ }^{51}$ Nevertheless, the stability observed in the 3- to 6-month period is troublesome. In nursing homes,

each resident must receive and the facility must provide the necessary care and services to attain or maintain the highest practicable physical, mental, and psychosocial well-being, in accordance with the comprehensive assessment and plan of care. ${ }^{52}$

In March 2009, changes to surveyor guidance provided in F-Tag 309 gave explicit direction about the nursing home's responsibility regarding effectively identifying specific pain type and pain management. ${ }^{53}$ Although data in the immediate post-implementation period suggested positive changes in response to the surveyor guidance with respect to recognition and management of pain, these findings did not extend to nursing home residents with severe cognitive impairment. ${ }^{54}$ If regulations and administrative initiatives do not meet the needs of residents who cannot report pain, research on how best to implement effective pain assessment and management strategies in this setting is warranted. Clinical implications include the need to ensure that pain in cognitively impaired populations is being managed adequately and recognition that the adjustment period after entering a nursing home and the unique pain and psychosocial needs of patients. Our findings of stable pain classes from 3 to 6 months indicate that this pain may not be adequately managed in this population.

\section{Strengths and Limitations}

We used a national data resource that included virtually all US nursing home residents. Doing so allowed for a large sample size of residents unable to self-report pain and provides a "real-world" look at the symptoms experienced by this population. Because we wanted our findings to be informative for all residents unable to self-report pain in nursing home settings, we did not limit our sample to specific populations likely to experience pain (eg, cancer or post-surgical populations). Our study provides a longitudinal evaluation of pain subgroups within the 
first 6 months of the nursing home stay. Previous crosssectional research has provided a snapshot of pain at admission, and only included residents with self-reported pain. ${ }^{55}$ Our study demonstrates that latent transition analysis is a useful tool to harness the power of longitudinal, high dimensional data for pain symptom science in an under-studied setting.

Our study is limited to the first 6 months of the nursing home stay, a critical time in the nursing home stay. Additional study is required to evaluate the extent to which our findings extend to longer stays. Evaluating pain subgroups throughout the entire nursing home stay may yield greater insights about the course of symptoms. Such insights may help develop processes of care to improve pain recognition and management. Our decision to focus on newly admitted residents with assessments at 3 and 6 months may have introduced selection bias as residents may have been excluded if they died or were discharged during the first 6 months. We recognize that studies using administrative data resources are limited to items collected. Our study lacked measures of specific domains of cognitive function. As recommended when using administrative data, ${ }^{56}$ we had a clear rationale regarding which indicators to include that was guided by a relevant conceptual model. ${ }^{19-22}$

\section{Conclusions}

Our longitudinal study supports the notion that pain subgroups may be differentiated by pain itself, depressive symptoms, and behavioral manifestations. Broadening the perspective pain expressions in those unable to self-report to include physical, behavioral, and psychological components may lead to better recognition of pain in nursing homes. Leveraging existing data and applying person-centered analytic approaches to identify residents in pain is an important first step towards improving pain recognition in those unable to voice their suffering. Algorithms to identify pain in residents unable to express their suffering coupled with pharmacological (eg, scheduled analgesics and/or adjuvants) and non-pharmacological (eg, massage, heating pad) treatments may improve pain management in nursing homes. ${ }^{57}$

\section{Key Message}

This article describes longitudinal changes in pain subgroups differentiated by physical functioning, depressive symptoms, and agitation for nursing home residents unable to self-report pain. Longitudinal changes in the pain subgroups over 6 months post-admission highlight that residents with severe cognitive impairment may be at risk for worsening pain.

\section{Funding}

This work was supported by the National Institutes of Health [NR016977 to K.L.L.], National Center for Advancing Translational Science [TR001454 to K.L.L.].

\section{Disclosure}

The authors report no conflicts of interest in this work.

\section{References}

1. Hunnicutt J, Ulbricht C, Tjia J, Lapane KL. Pain and pharmacologic pain management in long-stay nursing home residents. Pain. 2017;158:1091-1099. doi:10.1097/j.pain.0000000000000887

2. Dube C, Morrison R, Mack D, et al. Prevalence of pain on admission by level of cognitive impairment in nursing homes. J Pain Res. 2020. doi:10.2147/JPR.S270689

3. Ersek M, Nash P, Hilgeman M, et al. Pain patterns and treatment among nursing home residents with moderate-severe cognitive impairment. J Am Geriatr Soc. 2020;68:794-802. doi:10.1111/ jgs. 16293

4. Burfield A, Wan T, Sole M, Cooper J. Behavioral cues to expand a pain model of the cognitively impaired elderly in long-term care. Clin Interv Aging. 2012;7:207-223. doi:10.2147/CIA.S29656

5. Nowak T, Neumann-Podczaska A, Deskur-śmielecka E, Styszyński A, Wieczorowska-Tobis K. Pain as a challenge in nursing home residents with behavioral and psychological symptoms of dementia. Clin Interv Aging. 2018;25:1045-1051. doi:10.2147/CIA. S157246

6. Kunz M, Mylius V, Scharmann S, Schepelman K, Lautenbacher S. Influence of dementia on multiple components of pain. Eur J Pain. 2009;13:317-325. doi:10.1016/j.ejpain.2008.05.001

7. Warden V, Hurley AC, Volicer L. Development and psychometric evaluation of the Pain Assessment in Advanced Dementia (PAINAD) scale. J Am Med Dir Assoc. 2003;4(1):9-15. doi:10.1097/01. JAM.0000043422.31640.F7

8. Scherder EJ, Bouma A. Visual analogue scales for pain assessment in Alzheimer's disease. Gerontology. 2000;46(1):47-53. doi:10.1159/ 000022133

9. Hurley AC, Volicer BJ, Hanrahan PA, Houde S, Volicer L. Assessment of discomfort in advanced Alzheimer patients. Res Nurs Health. 1992;15(5):369-377. doi:10.1002/nur.4770150506

10. Kovach CR, Weissman DE, Griffie J, Matson S, Muchka S. Assessment and treatment of discomfort for people with late-stage dementia. $J$ Pain Symptom Manage. 1999;18(6):412-419. doi:10.1016/S0885-3924(99)00094-9

11. Feldh K. The checklist of nonverbal pain indicators. Pain Management Nursing, I. 2000;1:13-21. doi:10.1053/jpmn.2000.5831

12. Fisher SE, Burgio LD, Thorn BE, et al. Pain assessment and management in cognitively impaired nursing home residents: association of certified nursing assistant pain report, Minimum Data Set pain report, and analgesic medication use. $J$ Am Geriatr Soc. 2002;50 (1):152-156. doi:10.1046/j.1532-5415.2002.50021.x

13. Hadjistavropoulos T, Herr K, Prkachin K, et al. Pain assessment in elderly adults with dementia. Lancet Neurol. 2014;13:1216-1227. doi:10.1016/S1474-4422(14)70103-6

14. Strand L, Gundrosen K, Lein R, et al. Body movements as pain indicators in older people with cognitive impairment: a systematic review. Eur J Pain. 2019;23:669-685. doi:10.1002/ejp.1344 
15. Chopra K, Arora V. An intricate relationship between pain and depression: clinical correlates, coactivation factors and therapeutic targets. Expert Opin Ther Targets. 2014;18:159-176. doi:10.1517/ 14728222.2014.855720

16. Enache D, Winblad B, Aarsland D. Depression in dementia: epidemiology, mechanisms, and treatment. Curr Opin Psychiatry. 2011;24:461-472. doi:10.1097/YCO.0b013e32834bb9d4

17. Tse M, Wan V, Vong S. Health-related profile and quality of life among nursing home residents: does pain matter? Pain Manag Nurs. 2013;14:e173-e184. doi:10.1016/j.pmn.2011.10.006

18. Denkinger M, Lukas A, Nikolaus T, Peter R, Franke S. Multisite pain, pain frequency and pain severity are associated with depression in older adults: results from the ActiFE Ulm study. Age Ageing. 2014;43:510-514. doi:10.1093/ageing/afu013

19. Melzack R, Wall P. Pain mechanisms: a new theory. Science. 1965;150:971-978. doi:10.1126/science.150.3699.971

20. Novy D, Nelson D, Francis D, Turk DC. Perspectives of chronic pain: an evaluative comparison of restrictive and comprehensive models. Psychol Bull. 1995;118:238-247. doi:10.1037/0033-2909.118.2.238

21. Snow A, O'Malley K, Cody M, et al. A conceptual model of pain assessment for noncommunicative persons with dementia. Gerontologist. 2004;44:807-817. doi:10.1093/geront/44.6.807

22. Brant J, Beck S, Miaskowski C. Building dynamic models and theories to advance the science of symptom management research. $J$ Adv Nurs. 2010;66:228-240. doi:10.1111/j.1365-2648.2009.05179.x

23. U.S. Centers for Medicare and Medicaid Services. Long-Term Care Facility Resident Assessment Instrument 3.0 User's Manual, Version 1.14; 2016. Available from: https://downloads.cms.gov/files/MDS30-RAI-Manual-V114-October-2016.pdf. Accessed Oct.

24. U.S. Centers for Medicare and Medicaid Services. Centers for Medicare and Medicaid Services. Long Term Care Minimum Data Set (MDS) 3.0; Published 2011.

25. Lum TY, Lin WC, Kane RL. Use of proxy respondents and accuracy of minimum data set assessments of activities of daily living. J Gerontol a Biol Sci Med Sci. 2005;60(5):654-659. doi:10.1093/ gerona/60.5.654

26. Saliba D, Jones M, Streim J, et al. Overview of significant changes in the Minimum Data Set for nursing homes version 3.0. J Am Med Dir Assoc. 2012;13(7):595-601. doi:10.1016/j.jamda.2012.06.001

27. Thomas KS, Wysocki A, Intrator O, Mor V. Finding Gertrude: the resident's voice in Minimum Data Set 3.0. J Am Med Dir Assoc. 2014;15(11):802-806. doi:10.1016/j.jamda.2014.01.012

28. Saliba D, Buchanan J. Development and Validation of a Revised Nursing Home Assessment Tool: MDS 3.0. Centers Medicare Medicaid Serv. 2008.

29. Saliba D, DiFilippo S, Edelen MO, et al. Testing the PHQ-9 Interview and Observational Versions (PHQ-9 OV) for MDS 3.0. $J$ Am Med Dir Assoc. 2012;13(7):618-625. doi:10.1016/j. jamda.2012.06.003

30. Kroenke K, Spitzer RL, Williams JB. The PHQ-9: validity of a brief depression severity measure. J Gen Intern Med. 2001;16(9):606-613.

31. Kroenke K, Spitzer RL. The PHQ-9: a new depression diagnostic and severity measure. NJ: SLACK Incorporated Thorofare; 2002.

32. Kroenke K, Spitzer R, Williams J. The PHQ-9: validity of a brief depression severity measure. J Gen Inter Med. 2001;16:606. doi:10.1046/j.1525-1497.2001.016009606.x

33. Morris J, Fries B, Scaling MS. ADLs within the MDS. $J$ Gerontol a Biol Sci Med Sci. 1999;54:M546-553. doi:10.1093/gerona/54.11. M546

34. Katz S, Ford AB, Moskowitz RW, Jackson BA, Jaffe MW. Studies of illness in the aged: the index of ADL: a standardized measure of biological and psychosocial function. JAMA. 1963;185(12):914-919. doi:10.1001/jama.1963.03060120024016

35. Mahoney FI, Barthel DW. Functional evaluation: the Barthel Index: a simple index of independence useful in scoring improvement in the rehabilitation of the chronically ill. Md State Med J. 1965;14:61-65.
36. McCreedy E, Ogarek J, Thomas K, Mor V. The Minimum Data Set Agitated and Reactive Behavior Scale: measuring behaviors in nursing home residents with dementia. $J$ Am Med Dir Assoc. 2019;20:1548-1552. doi:10.1016/j.jamda.2019.08.030

37. Perlman CM, Hirdes JP. The aggressive behavior scale: a new scale to measure aggression based on the minimum data set. $J$ Am Geriatr Soc. 2008;56(12):2298-2303. doi:10.1111/j.1532-5415.2008.02048.x

38. Thomas KS, Dosa D, Wysocki A, Mor V. The Minimum Data Set 3.0 Cognitive Function Scale. Med Care. 2017;55(9):e68. doi:10.1097/ MLR.0000000000000334

39. Saliba D, Buchanan J, Edelen M, et al. MDS 3.0: brief interview for mental status. J Am Med Dir Assoc. 2012;13:611-617. doi:10.1016/j. jamda.2012.06.004

40. Morris J, Fries B, Mehr D, et al. MDS Cognitive Performance Scale. J Gerontol. 1994;49:M174-182. doi:10.1093/geronj/49.4.M174

41. Gruber-Baldini AL, Zimmerman SI, Mortimore E, Magaziner J. The validity of the Minimum Data Set in measuring the cognitive impairment of persons admitted to nursing homes. $J$ Am Geriatr Soc. 2000;48(12):1601-1606. doi:10.1111/j.1532-5415.2000.tb03870.x

42. Collins LM, Lanza ST. Latent Class and Latent Transition Analysis: With Applications in the Social, Behavioral, and Health Sciences. Hoboken, NJ: John Wiley \& Sons; 2013.

43. PROC LCA \& PROC LTA Users' Guide Version 1.3.2; 2015. Available from: https://www.methodology.psu.edu/files/2019/03/proc_lca_lta_ 1-3-2-1_users_guide-2ggq4d3.pdf. Accessed October 3, 2018.

44. Muthén LK, Muthén BO. Mplus User's Guide. Sixth Edition ed. Los Angeles, CA: Muthén \& Muthén; 1998-2011.

45. Gerrits M, van Oppen P, Leone S, et al. Pain, not chronic disease, is associated with the recurrence of depressive and anxiety disorders. BMC Psychiatry. 2014;14:187. doi:10.1186/1471-244X-14-187

46. Yuan Y, Min H, Lapane K, Rothschild A, Ulbricht C. Depression symptoms and cognitive impairment in older nursing home residents in the USA: a latent class analysis. Int $J$ Geriatr Psychiatry. 2020;35:769-778. doi:10.1002/gps.5301

47. Temkin-Greener H, Orth J, Conwell Y, Li Y. Suicidal ideation in US nursing homes: association with individual and facility factors. $A M$ J Geriatr Psychiatry. 2020;28:288-298. doi:10.1016/j. jagp.2019.12.011

48. Kales H, Gitlin L, Lyketsos C. When less is more, but still not enough: why focusing on limiting antipsychotics in people with dementia is the wrong policy imperative. J Am Med Dir Assoc. 2019;20:1074-1079. doi:10.1016/j.jamda.2019.05.022

49. Scott E, Kroenke K, Wu J, Yu Z. Beneficial effects of improvement in depression, pain catastrophizing, and anxiety on pain outcomes: a 12-month longitudinal analysis. $J$ Pain. 2016;17:215-222. doi:10.1016/j.jpain.2015.10.011

50. Pu L, Moyle W, Jones C, Todorovic M. Psychosocial interventions for pain management in older adults with dementia: a systematic review of randomized controlled trials. $J$ Adv Nurs. 2019;75:1608-1620.

51. Brownie S, Horstmanshof L, Garbutt R. Factors that impact residents' transition and psychological adjustment to long-term aged care: a systematic literature review. Int $J$ Nurs Stud. 2014;51:1654-1666. doi:10.1016/j.ijnurstu.2014.04.011

52. U.S Centers for Medicare and Medicaid Services. State operations manual: appendix PP - guidance to surveyors for long term care; Published 2017. Available from: https://www.cms.gov/Regulationsand-Guidance/Guidance/Manuals/downloads/som107ap_pp_guide lines_ltcf.pdf. Accessed November 1, 2020.

53. Planton J, Edlund B. Regulatory components for treating persistent pain in long-term care. J Gerontol Nurs. 2010;36:49-56. doi:10.3928/ 00989134-20100202-02

54. Lapane K, Quilliam B, Chow W, Kim MS. Impact of revisions to the F-Tag 309 surveyors' interpretive guidelines on pain management among nursing home residents. Drugs Aging. 2012;29:385-393. doi:10.2165/11599340-000000000-00000 
55. Ulbricht C, Hunnicutt J, Gambassi G, Hume A, Lapane K. Nonmalignant Pain Symptom Subgroups in Nursing Home Residents. J Pain Symptom Manage. 2019;57:535-544. doi:10.1016/j.jpainsymman.2018.11.023

56. Magee T, Lee S, Giuliano K, Munro B. Generating new knowledge from existing data: the use of large data sets for nursing research. Nurs Res. 2006;S50-56. doi:10.1097/00006199-200603001-00009
57. Reid M, Bennett D, Chen W, et al. Improving the pharmacologic management of pain in older adults: identifying the research gaps and methods to address them. Pain Med. 2001;12:1526-4637.

\section{Publish your work in this journal}

The Journal of Pain Research is an international, peer reviewed, open access, online journal that welcomes laboratory and clinical findings in the fields of pain research and the prevention and management of pain. Original research, reviews, symposium reports, hypothesis formation and commentaries are all considered for publication. The manuscript

Submit your manuscript here: https://www.dovepress.com/journal-of-pain-research-journa management system is completely online and includes a very quick and fair peer-review system, which is all easy to use. Visit http:// www.dovepress.com/testimonials.php to read real quotes from published authors. 